\title{
Der neue Markt
}

\author{
Erhard Taverna
}

In einer betriebswirtschaftlichen Diplomarbeit der Universität St. Gallen vom März 2000 wird über die strategische Positionierung eines Pharmakonzerns nachgedacht. Was am Fallbeispiel von Novartis exemplarisch demonstriert wird, sagt mehr über die Trends im Gesundheitsmarkt als alle einheimischen politischen Programme. Aus der Optik der Konzerne verschiebt sich das Gleichgewicht zum Endverbraucher, sprich Patienten, dessen Nachfragemacht im Verein mit Gesundheitsorganisationen Umsatz, Forschung und Marketing zunehmend beeinflusst. Die Versorgung wird im Cyberdialog interaktiv und individualistisch. Erst die Daueranalyse und der direkte Einbezug der Käufergruppen sichert dem Unternehmen die erwünschte Kundenloyalität. Weil die traditionelle Wertschöpfungskette der Gesundheitsversorgung in Europa auseinanderbreche, böten sich denjenigen Unternehmen neue Möglichkeiten, die integrierte Angebote hätten oder sich auf spezielle Nischen fokussierten.

\section{Das neue Team}

Im schönen Haus, in bester Wohnlage über dem See, verrät nur das kleine Schildchen über der Klingel, wem die vielen Computer gehören. Dominique Froidevaux, Frank Eicher und Viktor Hofmann bilden das Kernteam, ergänzt durch Reto Schumacher und Sandra Müller Buro. Zwei Mediziner, ein Ökonom, ein dipl. Ernährungsberater und eine Pharmazeutin konzentrieren ihre Erfahrungen in Health Care, Pharmamarketing, Informatik und Ökonomie seit Herbst 2001 in der eigenen Firma. Sie bieten marktorientierte Beratung für jeden Partner im Gesundheitswesen, kümmern sich um Marktanalyse und Projektumsetzung, entwickeln Konzepte für die Industrie und ärztliche Netzwerke und gestalten Websites für die Praxis.

Etwa im Auftrag des Produkteherstellers eine Datenbank mit einem Literaturservice für Onkologen oder ein Behandlungsprotokoll mit Statistik und Studienvergleich für das Arzneimittel einer anderen Firma. Zur Entwicklung gehören Applikationen für Marktstudien wie PEP, ein Patienten-Erfahrungs-Programm, das direkt die Erfahrung von Patienten nutzbar macht, oder ein Serviceportal für eine private Spitalunternehmung. Zum Programm gehören auch An- wenderbefragungen im Rahmen der Kundenforschung oder die Organisation von Arbeitsseminaren für Medikamentenverteilbetriebe.

Dem Ausblick über den sonnenbeschienen Balkon zum glitzernden See entspricht die gute Arbeitsatmosphäre in den funktional und doch freundlich eingerichteten Arbeitsräumen. Das Team fühlt sich wohl im expandierenden neuen Markt. An Ideen fehlt es nicht, die interdisziplinäre Arbeit macht Vergnügen und bringt die verschiedensten Auftraggeber an einen Tisch. Neue Ausbildungsmethoden für Pharmaassistentinnen oder elektronische Fortbildung für Ärzte werden aus eigenen Ressourcen oder in Zusammenarbeit mit spezialisierten Firmen ausgedacht und erprobt.

\section{Die neue Visitenkarte}

Ein Lieblingsprodukt ist die MyMedSite ${ }^{\mathrm{TM}}$ für Medizinalpersonen. Sie ist genau auf den neuen Konsumentenmarkt zugeschnitten, der über einen persönlichen Internetzugang verfügt. Mit dem Homepagegenerator ist die Praxiswebsite kein technisches Problem und dank einfacher Layoutauswahl, adaptierbarer Mustertexte und Coaching sogar kostengünstig. Der Inhaber kann seine neue Visitenkarte im geschützten Backoffice jederzeit selber neu- und umgestalten. Dem vorausgesagten, individualisierten Markt entspricht das Angebot von Ärztinnen und Ärzten, die sich von der Konkurrenz unterscheiden und profilieren wollen. Dabei lernen sie ganz nebenbei, was ein wirksamer Werbetext ist. Nützliche Informationen und attraktive Multimediaelemente sollen Patienten binden und neue anlocken. Wer einen Domainnamen reservieren lässt, baut seinen Vorsprung aus und macht seine Homepage erst recht zur einträglichen Wertschöpfungsquelle. Verpönt sind betrügerische promotionelle Aussagen, erlaubt sind aber Lauftexte wie «Der neue Grippeimpfstoff ist eingetroffen». Was bei uns konform ist, regeln die Richtlinien der FMH, die zum Beispiel vergleichende Werbung und Werbebanner, wie alles, was dem Berufsstand schaden könnte, nicht zulassen. Sponsoren dürfen erwähnt werden, wenn dies nach den Regeln von HON, Health On the Net, geschieht. Die Organisation mit Sitz in Genf wacht mit Benimm-dich-Regeln über den neuen 
Boom und belohnt ihre Validierung mit der Erteilung eines Gütesiegels. Vorläufig noch wird das Institut vom Standortkanton und von der Universität finanziert.

Ein oft zitiertes Bonmot sagt: «Der Patient steht für uns im Mittelpunkt. Doch da steht er im Weg.»?? Mit zunehmender Information und
Transparenz steigen zwar seine Wahlmöglichkeiten und sein direkter Einfluss auf Entscheidungsprozesse im Gesundheitswesen, gleichzeitig verschärft sich die Qual der Wahl. Im neuen Markt werden nicht nur die traditionellen Rollen, sondern auch die Rechte und Pflichten neu zu verteilen sein.

\section{Geschichte eines Dramas}

\section{F. Fischer}

\section{Das reale Drama}

Von 1997 bis 2000 arbeitete ich als Assistenzarzt in einer psychiatrischen Klinik in der Innerschweiz. Diese Klinik wurde von ihren ursprünglichen Eigentümern, einem überalterten katholischen Frauenorden, an einen von der wunderschönen Liegenschaft faszinierten Bauunternehmer aus Brugg (AG) verkauft, der in der Folge die Klinik rigoros umstrukturieren liess, Chefarzt und Pflegedienstleiter von heute auf morgen freistellte und Mitarbeiter/innen und Patienten/ -innen verunsicherte, so dass es zu zahlreichen Kündigungen, gegenseitigem Misstrauen und mit einer in der Klinikgeschichte noch nie dagewesenen Häufung von Suiziden einherging. Ein Artikel mit dem Titel «Schnäppchenjäger», erschienen im Magazin des Züricher Tages-Anzeigers im September 2000, beschrieb die damalige Situation, zumindest aus meiner Sicht, sehr treffend.

\section{Das literarische Drama}

Protestschreiben und Petitionen angesichts der kaum noch verantwortbaren Situation an die neue Leitung brachten keine Veränderungen. Um nicht nur ohnmächtig den Kopf schütteln zu müssen, fing ich damals an, die Situation für mich in Theaterszenen zu verarbeiten. Die ersten Entwürfe dienten vor allem der Psychohygiene, meiner wie auch der der Kollegen. Dann aber hatte ich Gelegenheit, über einen vom MigrosKulturprozent gesponserten Workshop des Internationalen Theaterinstituts (ITI) die Szenen in ein bühnentaugliches Stück umzuarbeiten. An- geleitet wurde ich dabei von erfahrenen Dramaturgen (Rainer Hofmann, Theater Winkelwiese/ Theater Neumarkt; Carl Hegemann, Berliner Volksbühne), die mir halfen, mich in der Neufassung von den konkreten Ereignissen zu lösen und die Turbulenzen von Betriebsübernahmen ganz allgemein aufs Korn zu nehmen.

Aus der psychiatrischen Klinik wurde eine Kathedrale, aus dem Chefarzt der Küster. Therapeuten wurden zu Organisten. Statt Patienten wurden nun Touristen gemolken, die man organisiert durch die Kathedrale schleuste, perfekte Hotellerie inklusive. In der Aushöhlung einer heilkräftigen Madonnenstatur, der «Schwarzen Madonna», wurden Werte ausgehöhlt und, stückchenweise in Kunstharzblöcke gegossen, profitabel vermarktet. Lohnkürzungen gab es hier wie dort, es wurde umstrukturiert, gekündigt, hintertrieben und gelogen, dass sich die Balken bogen, skurrile Menschen verloren ihre Nischen und dekompensierten tödlich, andere fanden im veränderten Hierarchiegefüge ihre lang vermisste Chance.

Aus der Dokumentation einer Tragödie war eine Groteske entstanden, die auf die Grenzen der Privatisierung im Gesundheitswesen hinweist. Vor allem die Psychiatrie ist hier ein sehr sensibler Bereich, den man nicht einfach zahlungskräftigen Investoren überlassen darf. Was bei der Fabrikation von Kühlschränken behebbar sein mag, nämlich Qualitätseinbussen durch Rationalisierung, lässt in der Psychiatrie die Suizidrate steigen.

Es ist nicht einfach, ein Theaterstück zu schreiben, noch schwieriger ist es, es auf die Bühne zu bringen. Ich bot mein Stück also mehreren Regisseuren an, die durchweg ablehnend 
antworteten, wenn auch mit sehr ausführlicher konstruktiver Kritik. Auch direkt angefragte Bühnen waren nicht interessiert. Erst der Gang zum teaterverlag ellg, der inzwischen auch die Rechte am Stück erworben hat, brachte den richtigen Kontakt. Thomas Senn, Regisseur der Hottwiler Spielleute, arbeitete das Stück in eine gestraffte Mundartfassung um und inszenierte es mit seiner Truppe in Hottwil, 11 km von Brugg entfernt, dem Wohnort des Investors. So wurde sogar der von mir erhoffte lokale Bezug noch Wirklichkeit.

Bei der ersten Probe, bei der ich dabei sein dufte, war ich schlichtweg überwältigt. Die eigenen Gedankenwelten plötzlich konkret vor Augen zu sehen und gleichzeitig emotional wieder mitten in den Ereignissen zu stehen. Von der Zusammenarbeit mit dem Regisseur konnte ich viel lernen. Ich war von den Leistungen der Schauspieler beeindruckt, von der technisch aufwendigen Inszenierung wie auch vom Interesse des Publikums. Die sieben Aufführungen im März 2002 vor je 200 Zuschauern waren alle ausverkauft.

Der Erfolg der Aufführungen und die Anerkennung der ehemaligen Kollegen waren für mich eine Art späte «Rehabilitation». Über die Aufführung bekam die andere Sicht noch einmal Öffentlichkeit und Zustimmung. "Wahrheit» ist sehr relativ und flexibel. Wenn, wie der neue Manager der Klinik so schön formuliert hat, «mindestens 50\% der Belegschaft ausgewechselt sind», dann hat man oben endlich Ruhe, dann lassen sich Zusammenhänge neu darstellen, dann fehlen die Menschen, die alte Fakten noch in Erinnerung hatten und konträre Meinungen vertraten.

In den Monaten vor meiner Kündigung geriet ich in zunehmende innere Spannung. Was da bei Betriebsversammlungen salbungsvoll verkündet wurde, war meist das Gegenteil von dem, was ich selber wahrnahm und mitbekam. Die ständigen Beteuerungen von oben her wurden zwar mit jeder Wiederholung unglaubwürdiger, trotzdem wuchs auch der Zweifel an der eigenen Meinung, die nur noch im engen Kollegenkreis ihren Platz behielt. Dies umso mehr, je mehr andere Mitarbeiter bei offiziellen Verlautbarungen schwiegen oder verhalten mitnickten. Die Aufführung des Theaterstücks gab mir das Gefühl zurück, damals «richtig» empfunden zu haben.

\section{Das gesellschaftliche Drama}

Zum Schluss noch ein für mich sehr eindrucksvolles Erlebnis: Im Anschluss an eine Aufführung wurde ich von einem interessierten Ehepaar über die Hintergründe des Stücks befragt. Der Mann realisierte daraufhin, dass er just von dieser Gesellschaft, die in der Dramaturgie des eben gesehenen Dramas federführend war, eine beträchtliche Anzahl Aktien besitzt und berichtete über die kürzlich stattgefundene Hauptversammlung. Dort sei von internen Schwierigkeiten der Klinik die Rede gewesen, aufgrund deren man Personal habe auswechseln müssen. Jetzt aber sei die Klinik auf gutem Kurs, mit baldigen schwarzen Zahlen werde gerechnet. Von Suiziden keine Rede.

Der Mann war betroffen darüber, dass er selber von Entwicklungen in der Wirtschaft, so wie wir alle, profitiert, ohne sich bewusst zu sein, was das jeweils in der Kehrseite für einzelne bedeuten kann. Hier fallen reihenweise Menschen, vorher noch integriert und halbwegs kompensiert, bei Rationalisierungsprozessen mangels Flexibilität aus ihren Nischen, werden depressiv, somatisieren, brauchen IV-Renten und Sozialhilfe und kosten die Volkswirtschaft ein Vielfaches von dem, was die Firmen und Aktionäre einstreichen. Von den Kränkungen und dem menschlichen Leid der Betroffenen gar nicht zu reden.

Das ist das eigentliche Drama.

Fischer F. Gute Männer kommen in den Himmel. Belp: teaterverlag elgg; 2000. 